TITL: DISORDER-INDUCED BREAKDOWN OF SOLITON AND POLARON PARTICLES

AUTHOR(S): A. R. Bishop

David Cai

N. Grønbech-Jensen

M. I. Salkola

SUBMITTED TO: Proceedings

"Fluctuation Phenomena: Disorder and Nonlinearity",

Madrid, Spain, September 27 - October 1, 1994

By acceptance of this article, the publisher recognized that the $U \mathrm{~S}$ Government retains a nonexclusive, royalty-free license to publish or reproduce the published form of this contribution or to allow others to do so for US Government purposes.

The Los Alamos National Laboratory requests that the publisher identily this article as work performed under the auspices of the U S Department of Energy.

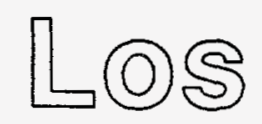

A4ロ

FORM NO. 836 R4

ST. NO. 2629 5/81
Los Alamos National Laboratory Los Alamos, New Mexico 87545 MASTER

DISTRIBUTION OF THIS DOCUMENT IS UNLIMITED 


\section{DISCLAIMER}

Portions of this document may be illegible in electronic image products. Images are produced from the best available original document. 


\title{
DISORDER-INDUCED BREAKDOWN OF SOLITON AND POLARON PARTICLES
}

\author{
A.R. Bishop, David Cai, N. Grønbech-Jensen and M.I. Salkola \\ Theoretical Division and Center for Nonlinear Studies, \\ Los Alamos National Laboratory, Los Alamos, NM 87545, U.S.A.
}

\begin{abstract}
Using examples of the perturbed $(1+1)$ dimensional sine-Gordon, the continuous and discrete nonlinear Schrödinger systems, and a three-site quantum polaron problem, we briefly review some phenomena related to the fascinating interplays between nonlinearity, disorder, noise, nonadiabaticity, and lattice discreteness. The concept of competing length-scales and time-scales is emphasized as they pertain to the common concept of solitons and polarons behaving as "particles".
\end{abstract}

\section{Introduction}

The evolving understanding of "nonlinearity with disorder" is revealing fascinating interplays of several key physical ingredients: Nonlinearity, Disorder, Noise (thermal, quantum, and otherwise), Nonadiabaticity, and Lattice Discreteness. A dominating feature of these considerations is the role of "competing spatial and temporal scales", which leads to a variety of complex phenomena. To illustrate some of these phenomena, we describe here three model systems:

(i) Parametric disorder in the $(1+1)$ sine-Gordon (SG) and nonlinear Schrödinger (NLS) equations. We summarize (a) length-scale competitions which determine the stability of pre-existing breathers (solitons), and (b) a competition between nonlinear self-focusing (collapse), and linear (Anderson) localization in disordered NLS with spatially extended initial data, which controls soliton formation.

(ii) Discrete $(1+1)$ NLS equations, emphasizing integrable versus nonintegrable systems and some qualitative effects of lattice discreteness - the possibility of "staggered" solitons, and a nonlinear generalization of Bloch oscillations and dynamic localization in an external electric field. We also discuss the importance of nonintegrability for the distinction between recurrence and stochasticization.

(iii) Quantum polarons in a simple three-site electron-phonon cluster, which serves to (a) reveal important nonlinear and generally nonadiabatic effects in expermentally relevant quantities (optical, vibrational, dynamic correlation functions), and (b) the effect of disorder on the localization of particle-like coherence of polarons.

\section{DISCLAIMER}

This report was prepared as an account of work sponsored by an agency of the United States Government. Neither the United States Government nor any agency thereof, nor any of their employees, makes any warranty, express or implied, or assumes any legal liability or responsibility for the accuracy, completeness, or usefulness of any information, apparatus, product, or process disclosed, or represents that its use would not infringe privately owned rights. Reference herein to any specific commercial product, process, or service by trade name, trademark, manufacturer, or otherwise does not necessarily constitute or imply its endorsement, recommendation, or favoring by the United States Government or any agency thereof. The views and opinions of authors expressed herein do not necessarily state or reflect those of the United States Government or any agency thereof. 


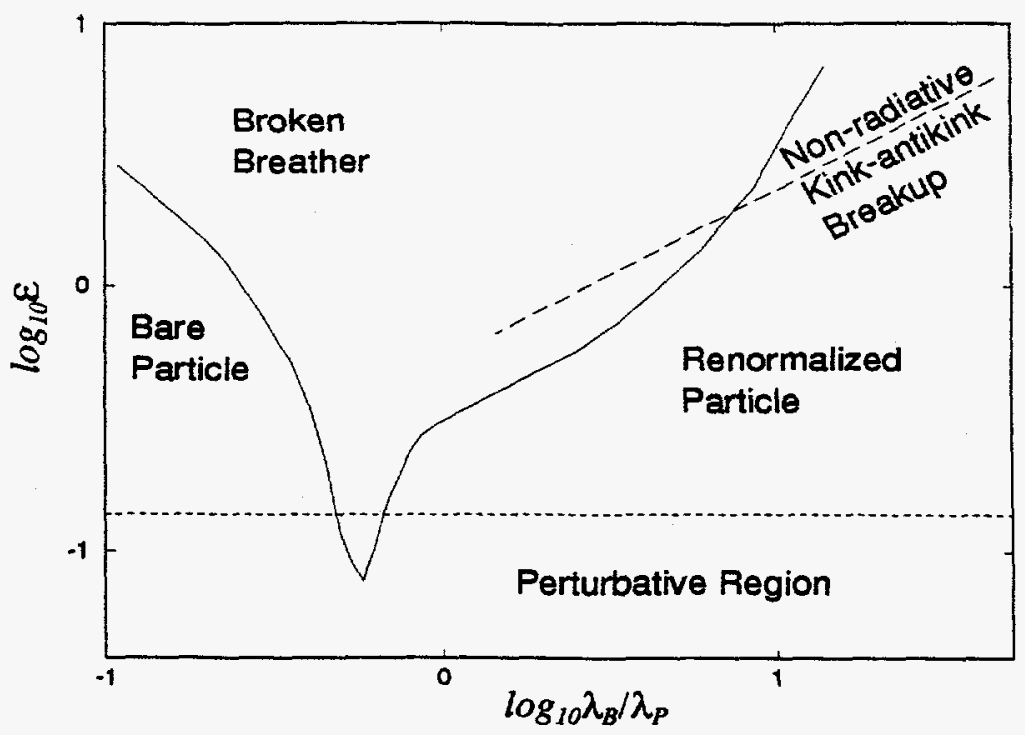

Figure 1: Schematic "phase diagram" (see text).

\section{Parametric Perturbations}

In a series of recent studies ${ }^{1-5}$, we have extensively examined the $(1+1)$-dimensional SG,

$$
U_{t t}-U_{x x}+[1+V(x)] \sin U=0,
$$

and NLS,

$$
i \psi_{t}+\psi_{x x}+2 \psi|\psi|^{2}=V(x) \psi
$$

equations where the parametric perturbation $V(x)$ can take a variety of forms - local impurities, a uniform or periodically oscillating ramp, spatially periodic or quasiperiodic, random, periodic impulses, etc.

The case of a periodic perturbation, $V(x)=\epsilon \cos (k x)$, is particularly important. The stability of a breather (soliton) is summarized schematically in Fig. 1 - numerical details can be found for SG and NLS in Refs. 1-3 and 4, 5, respectively. This figure captures a number of essential features.

First, in a nonlinear system, we need to specify more than one scaling function. As a minimum, here we see that both the ratios of the amplitudes and characteristic lengths of the perturbation and coherent nonlinear structure are "relevant". As the perturbation becomes stronger it breaks the nonlinear structure into a series of disconnected distortions, ultimately described as linearly localized states, i.e., the linear, disordered fixed-point. On the other hand, as the unperturbed breather width $\lambda_{B}$ becomes large compared to the perturbation period $\lambda_{P}$, it smooths away the perturbation influence, i.e., leading to the nonlinear, ordered fixed point.

Second, at moderate perturbation strength but where $\lambda_{B} / \lambda_{P} \gtrsim 1$ or $\lesssim 1$, classical collective coordinate perturbation theory is analytically very successful (indeed para- 

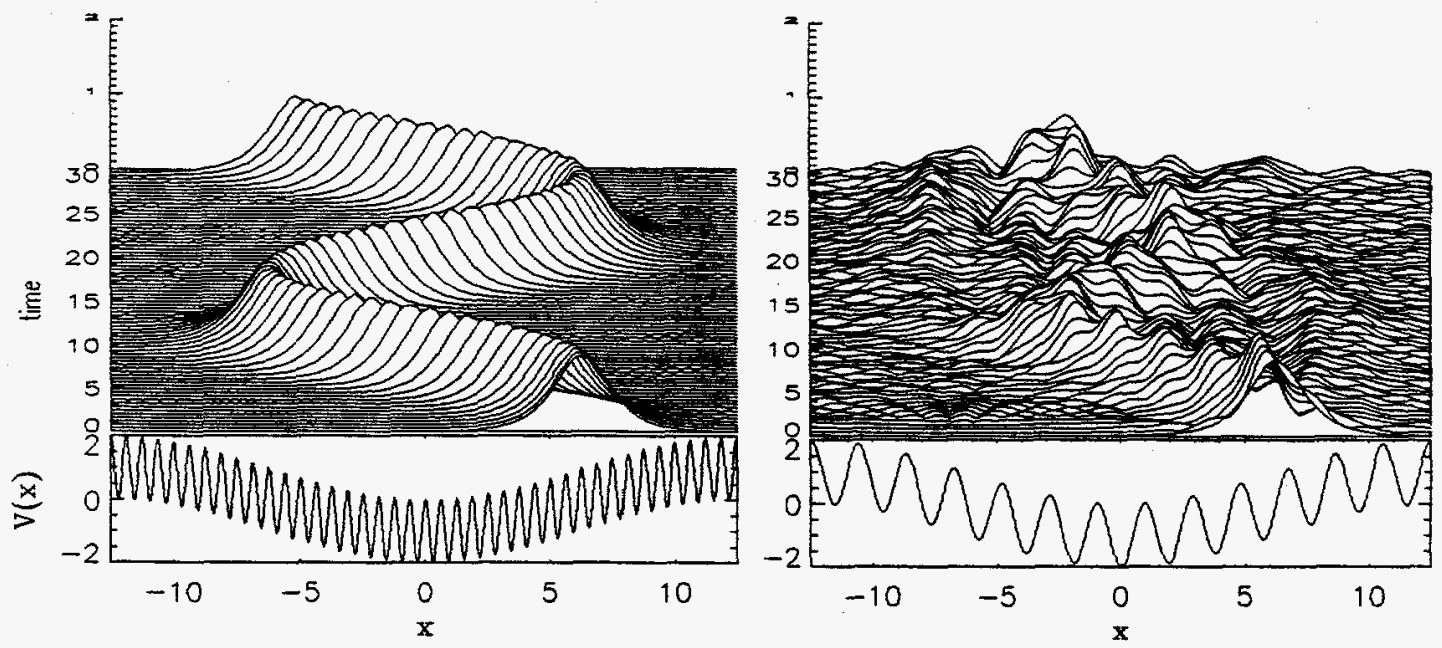

Figure 2: The external parametric potential has the form $V(x)=\epsilon_{1} \cos \left(2 \pi p_{1} x / L\right)+$ $\epsilon_{2} \cos \left(2 \pi p_{2} x / L\right), \epsilon_{1}=\epsilon_{2}=1$. Left: The renormalized particle limit, $p_{1}=1, p_{2}=40$; Right: Destruction of the soliton, $p_{1}=1, p_{2}=13$.

metric perturbations are much gentler than additive ones ${ }^{1-3}$ in this regard), i.e., the breather (soliton) behaves substantially as a coherent "particle".

Third, even for weak perturbation strengths, when $\lambda_{B} \sim \lambda_{P}$ there is a highly destructive "length-scale" competition, which leads to the rapid decay of the soliton. Here, coherent solitons and nonlinear mode reduction are of little value. The system reveals its particle-radiation duality directly: The decay is through the emission of radiation (phonons). In fact, a very related length-scale competition occurs for topological kink-solitons in the periodically perturbed SG equation and the conditions for phonon emission can be understood in a similar way ${ }^{3}$, namely the overlap of the linear perturbation spectrum with the phonons - as the velocity of the soliton increases, this reduces the phonons available for emission (the minimum in Fig. 1 is less pronounced), and additional resonances appear as a function of the propagation velocity ${ }^{3}$.

The periodic perturbation is especially important because it demonstrates the role of color in the disorder. This is shown again in Fig. 2 for an NLS case, where a perturbation with two periods is used. We see that the particle character is preserved in the long-wavelength component but destroyed by the shorter-wavelength one when it competes with the soliton width. For a generally colored perturbation, the soliton moves as a "particle" (in a bare or renormalized perturbation for $\lambda_{B}\left\langle\lambda_{P}, \lambda_{B}\right\rangle \lambda_{P}$, respectively) except for those occasional spatial regions where (locally) $\lambda_{B} \sim \lambda_{P}$. This insight allows us to construct a transport phenomenology for solitons as particles in a disordered medium if we can understand the re-focusing of radiation into solitons 


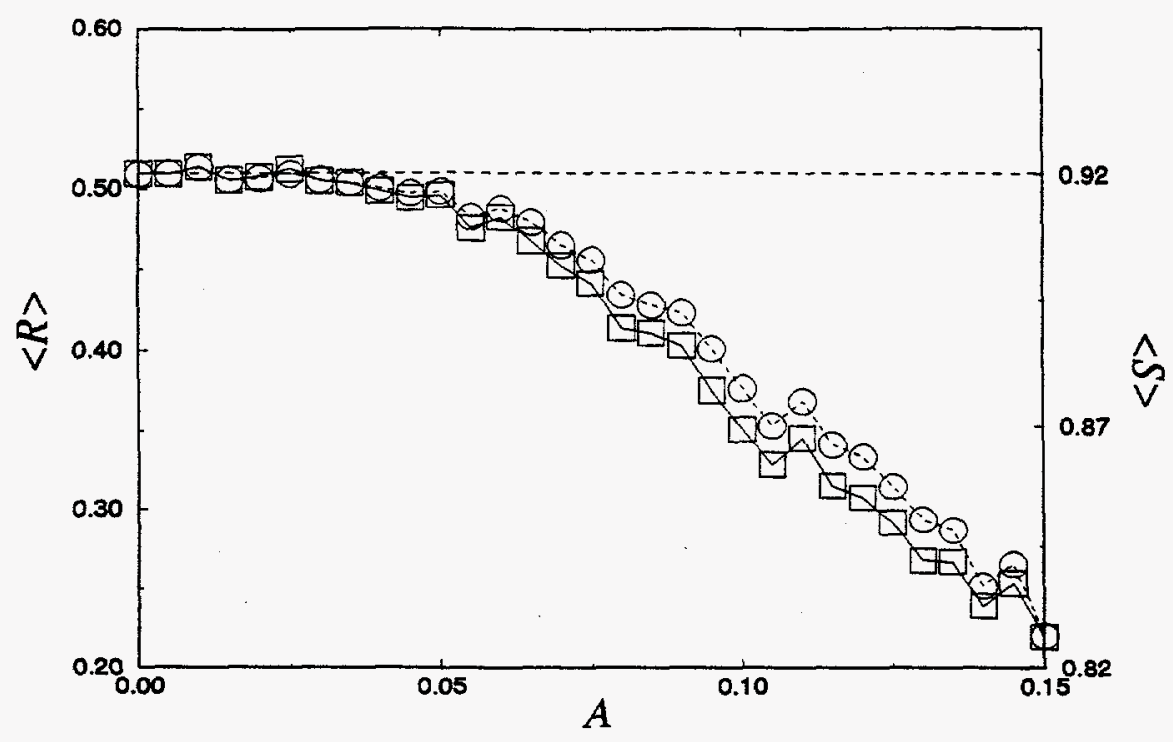

Figure 3: Localization in a disordered NLS. The square is the averaged participation ratio $\langle R\rangle$, and the circle is the averaged entropy $\langle S\rangle .\langle\cdots\rangle$ is the average over the simulation time $t=10000$ (time units) and 20 realizations of the disorder. The axes of the quantities have been scaled to show their similarity in measuring the localization. The dashed line is the participation ratio (entropy) for the linear Anderson localization. $A$ is the amplitude of the initial wavefunction, i.e., $\psi_{n}(0)=A$.

after the occasional destructions.

As a step to understanding this last issue, namely the formation of solitons, we have considered the case summarized in Fig. 3. Here, the discrete NLS equation with a flat initial condition of amplitude $A$ is subjected to parametric disorder of a form which is familiar from Anderson (linear) localization theory, namely the siteuncorrelated probability distribution $P\left(V_{n}\right)$, with $P$ the normalized flat-top distribution in the interval $-V_{m} \leq V \leq V_{m}$. Very long-time simulations $(t=10000$ time units) on long chains $(N=200)$, averaged over 20 realizations of disorder were completed. The results are monitored by measuring the participation ratio $R$

$$
R=\frac{\sum_{n=1}^{N}\left|\psi_{n}\right|^{2}}{N \sum_{n=1}^{N}\left|\psi_{n}\right|^{4}}
$$

or the entropy $S$

$$
S=-\frac{1}{\ln N} \sum_{n=1}^{N} p_{n} \ln p_{n},
$$




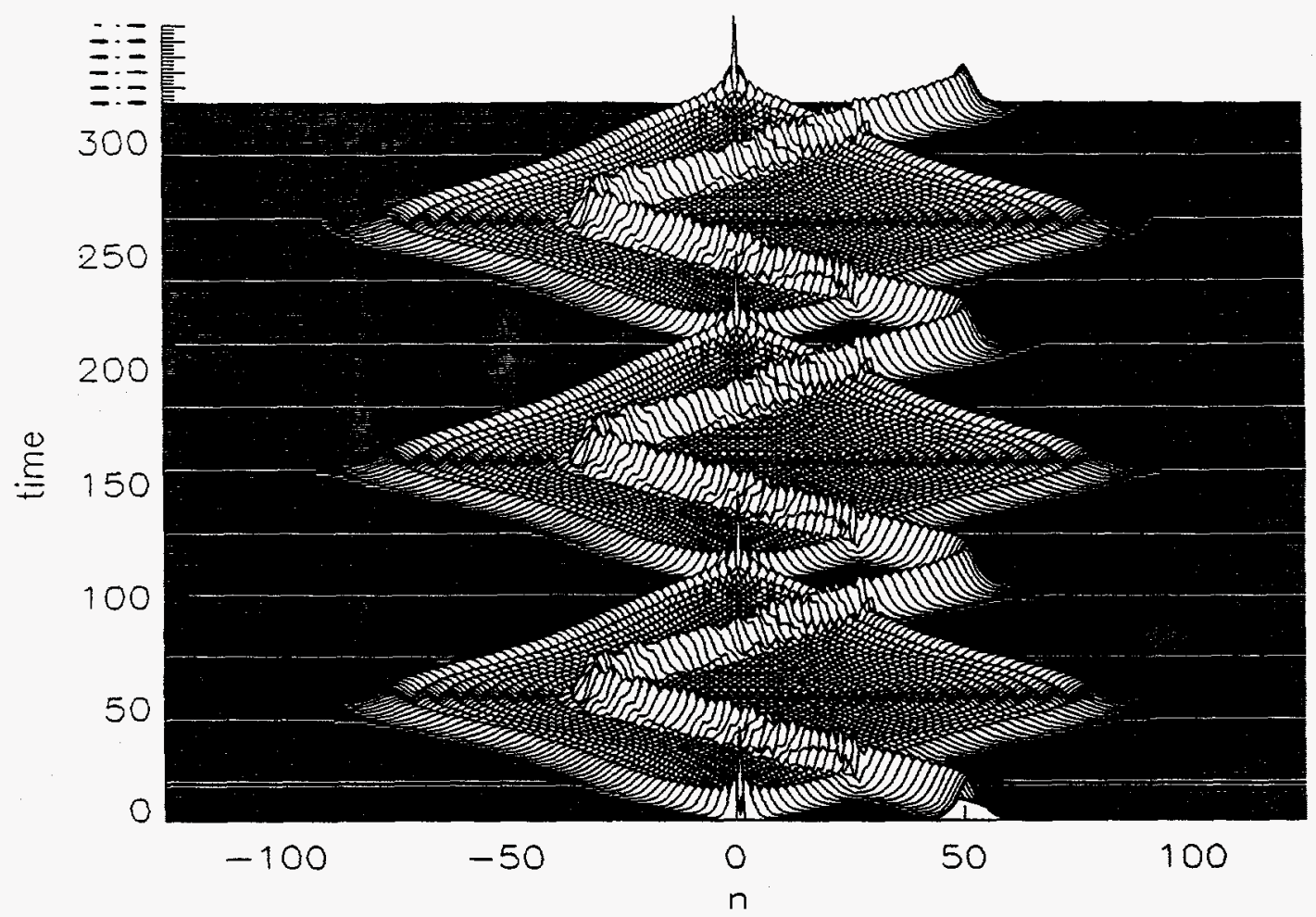

Figure 4: Evolution of the system (6) with $\mu=1$ and $\nu=0$ (integrable) at the resonance condition $\mathcal{E} / \omega=x_{1}$, where $x_{1}$ is the first zero of the Bessel function $J_{0}(x)$ and $\omega=0.06$. Plotted here is $\left|\psi_{n}(t)\right|$.

where

$$
p_{n}=\frac{\frac{1}{\mu} \ln \left(1+\mu\left|\psi_{n}\right|^{2}\right)}{\sum_{n=1}^{N} \frac{1}{\mu} \ln \left(1+\mu\left|\psi_{n}\right|^{2}\right)} .
$$

Fig. 3 shows the situation for $V_{m}=0.3$. We see that, for amplitude $A \leqslant A_{c}=0.03$, the localization is as expected for a disordered linear Schrödinger equation: here, the disorder prevents the focusing (modulational instability) which is characteristic of the unperturbed NLS; the disorder induces linear localization and the nonlinearity is never felt. However, for $A \gtrsim A_{c}=0.03$, the nonlinear modulational instability dominates; as self-focusing evolves, the disorder smoothing discussed earlier occurs and the nonlinearity dominates, leading to increasingly localized NLS solitons as the available energy increases (through the increasing amplitude $A$ ). Thus, again, the linear, disordered and nonlinear, ordered fixed-points describe much of the behavior. Clearly, $A_{c}$ increases monotonically from zero as $V_{m}$ increases from zero.

It is important to note one additional feature of Fig. 3. The crossover region $A \sim A_{c}$ is not sharp. This is an intrinsic property of the nonlinear disordered system. Although we can understand the $A \lesssim A_{c}$ and $A \gtrsim A_{c}$ regimes quite well by 


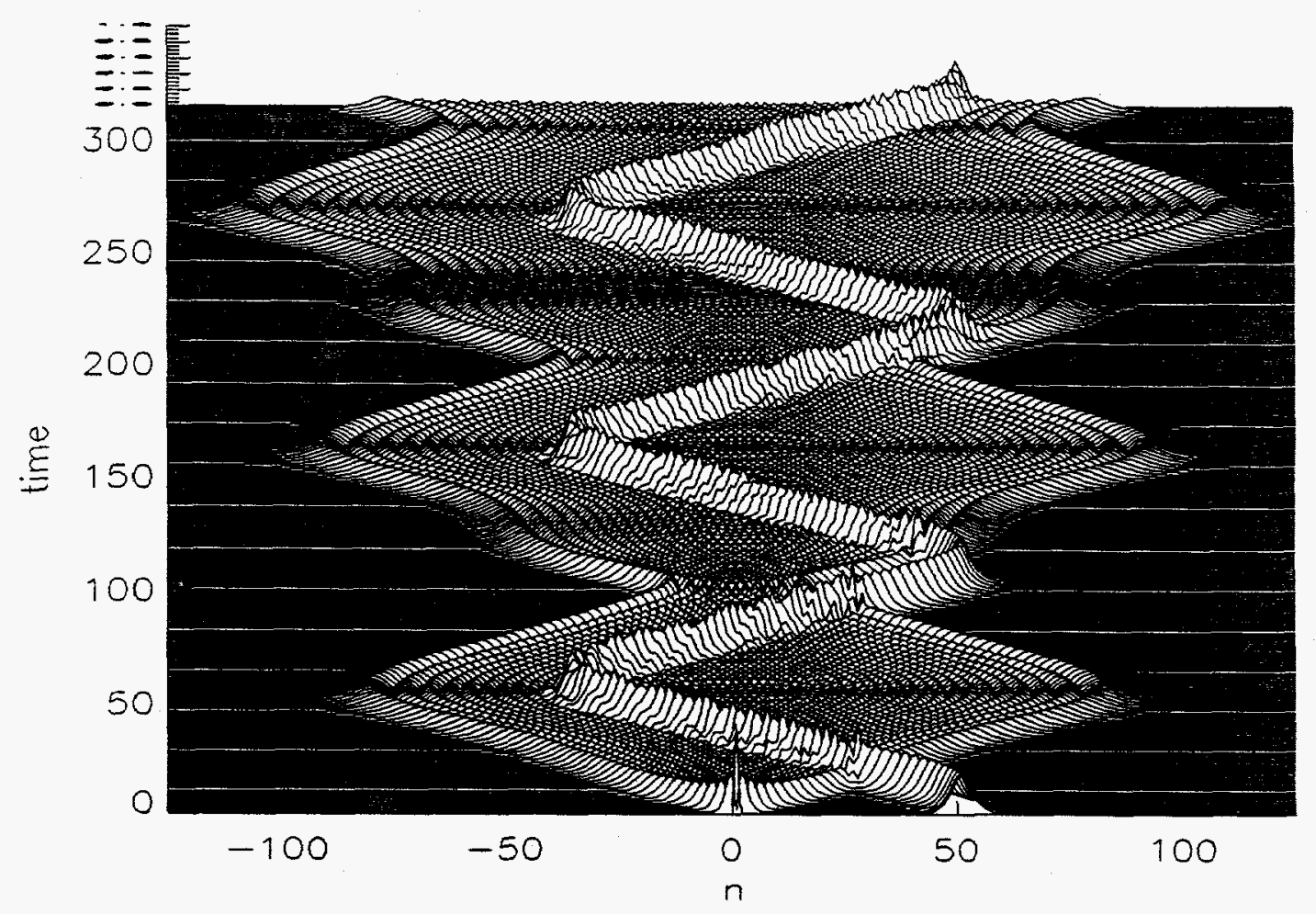

Figure 5: Evolution of the system (6) (integrable) off resonance condition. The parameter are the same as Fig. 4 except that $\mathcal{E} / \omega=0.9 x_{1}$.

invoking stationary phase ansätze - for the linearly localized and unperturbed NLS soliton, respectively, the fluctuations in the region $A \sim A_{c}$ are intermittent transitions between these two stationary-phase attractors. This is an important phenomenon, reflecting the fact, unlike the situation for the linear Schrödinger equation with stationary noise, the time evolution is not necessarily captured by a stationary phase state which slaves frequency and energy - the solution space is larger, and is visited in the crossover region here, as it was in the length-scale competition regimes of Figs. 1 and 2.

\section{Discrete NLS Equations}

Many physical systems are intrinsically discrete in space because of an underlying lattice or periodically modulated parameters (e.g., by nanoscale engineering). In this context, we must also consider the interplay with integrability and it is fortunate that a generalized discrete NLS system is possible which smoothly interpolates between an integrable limit (the Ablowitz-Ladik system ${ }^{6}$ ) and the standard nonintegrable discretization of the cubic nonlinearity. This system is

$$
i \dot{\psi}_{n}=-\left(\psi_{n+1}+\psi_{n-1}\right)-\left[\mu\left(\psi_{n+1}+\psi_{n-1}\right)+2 \nu \psi_{n}\right]\left|\psi_{n}\right|^{2}
$$




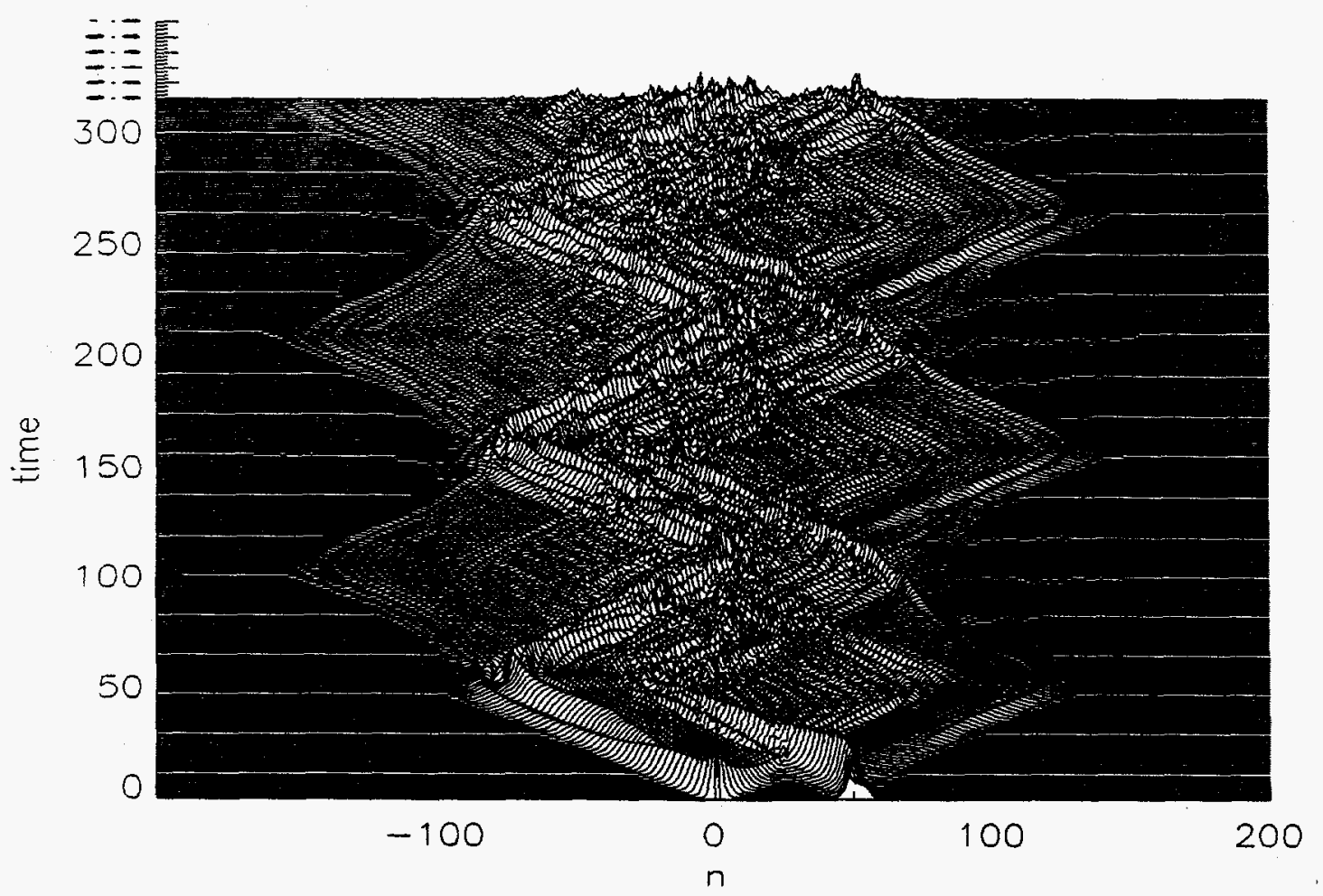

Figure 6: Evolution of the system (6) with $\mu=0$ and $\nu=1$ (nonintegrable) at $\mathcal{E} / \omega=x_{1}$, $\omega=0.06$. (cf. Fig. 4)

For $\nu=0$, this is the integrable Ablowitz-Ladik system. The general model has a well-defined Hamiltonian structure ${ }^{7,10}$ and has also been studied in the context of quantization $^{8}$. Interestingly, an electrical network can also be described by the above equation ${ }^{9}$.

We mention here three discrete lattice properties which are conveniently studied with system (6).

First, a discrete lattice results in a linear mode dispersion relationship which is bounded at high as well as low frequency (unlike the continuum Debye spectrum). This allows the possibility of breather-like phonon bound states with frequencies above as well as below the phonon band. The nonlinear modes above the band, however, have the form of "staggered" patterns, alternating phase from site to site, since they inherit their parentage from the $k=\pi / a$ ( $a=$ lattice constant) zone edge phonon. They are in fact prototypical examples of the "localized oscillatory" states (gap states) introduced in some generality by Takeno ${ }^{11}$ and others. They are known analytically in the integrable $(\nu=0)$ limit of Eq. 6 and play an important role in the dynamics of the discrete NLS in the presense of impurities etc. ${ }^{12}$. Their behavior has been studied in Ref. 7, where it is shown that they are stable in the "dark soliton" case and may be viewed as "particles" with negative effective mass. These particles move in a periodic Peierls-Nabarro potential, which can be analytically approximated ${ }^{7,10}$. 


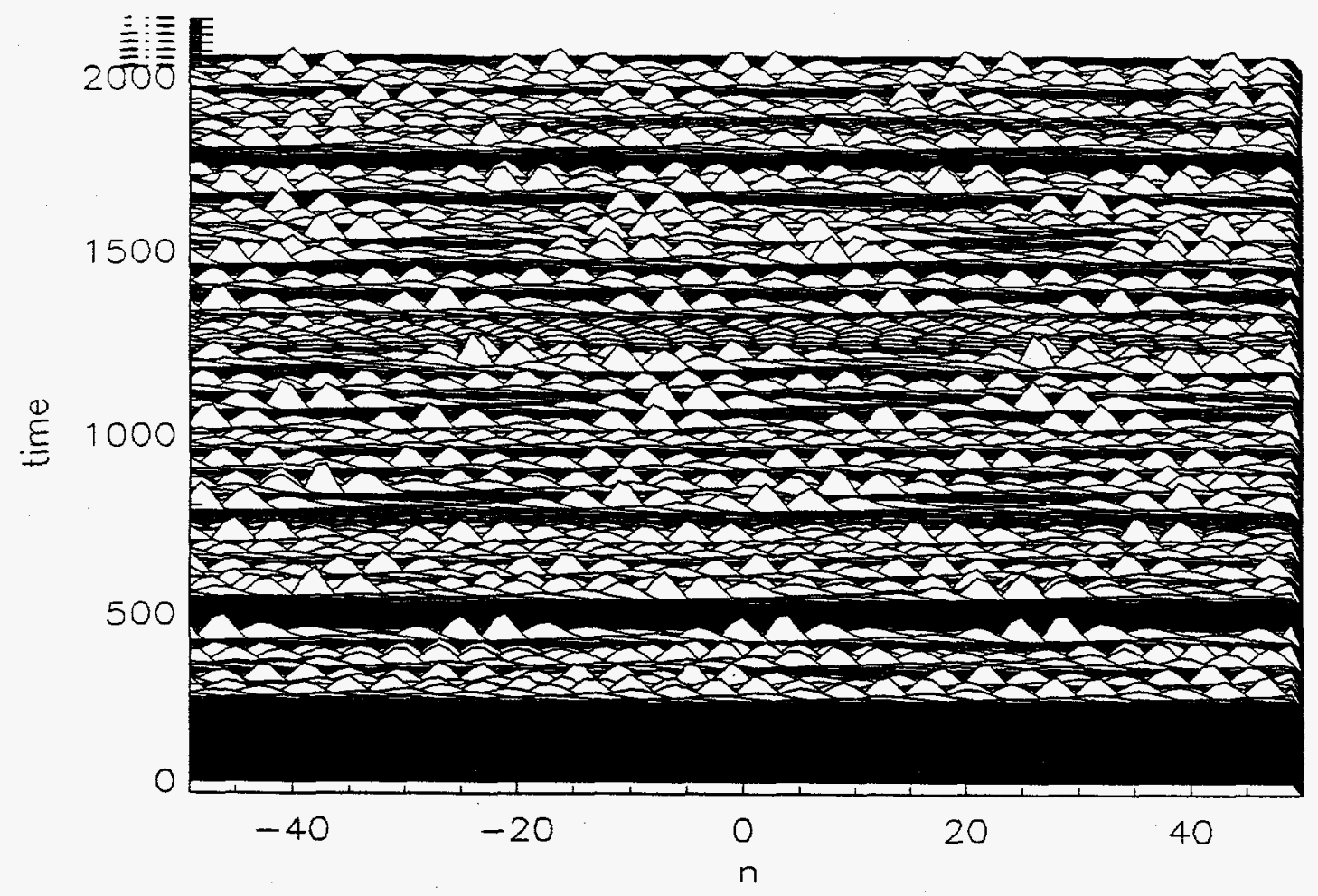

Figure 7: Recurrence: Evolution of the system (6) with $\mu=1$ and $\nu=0$ (integrable). The "black bands" appear when the system returns to the initial condition. $\left|\psi_{n}(t)\right|$ is plotted here.

The second discrete lattice effect is exhibited by considering the time-dependent perturbation

$$
n f(t) \psi_{n}
$$

Interestingly, this uniform ramp perturbation preserves the integrability of the $\nu=0$ Ablowitz-Ladik system, in the same way as for the continuum NLS ${ }^{13}$. The exact solution for arbitrary $f(t)$ and initial conditions can be explicitly constructed by, e.g., nonlinear spectral transform techniques ${ }^{10}$, as has been noted independently in Ref. 14. The case of $f(t)=$ constant was considered originally in Ref. 15 and more recently in Ref. 12, exhibiting bounded oscillatory soliton motion ${ }^{15,12}$ - unlike the continuum limit, where the soliton particle runs down the ramp. This bounded motion is a nonlinear extension of the Stark effect, and is a direct consequence of the lattice discreteness as in the linear limit ${ }^{17}$. Technically, it is described perfectly for a single soliton by a collective coordinate reduction in which the discrete lattice energy and norm properties of the Ablowitz-Ladik system are invoked ${ }^{12}$. The case of $f(t)=$ $\mathcal{E} \cos (\omega t)$ is equally interesting, providing an example of nonlinear Bloch oscillations and dynamical localization ${ }^{16}$. This is illustrated in Fig. 4, where the evolution of an initial condition comprised of a soliton plus a $\delta$-function is displayed. As in the analogous linear problem, a series of parametric resonance frequencies appears, $\mathcal{E} / \omega=$ 


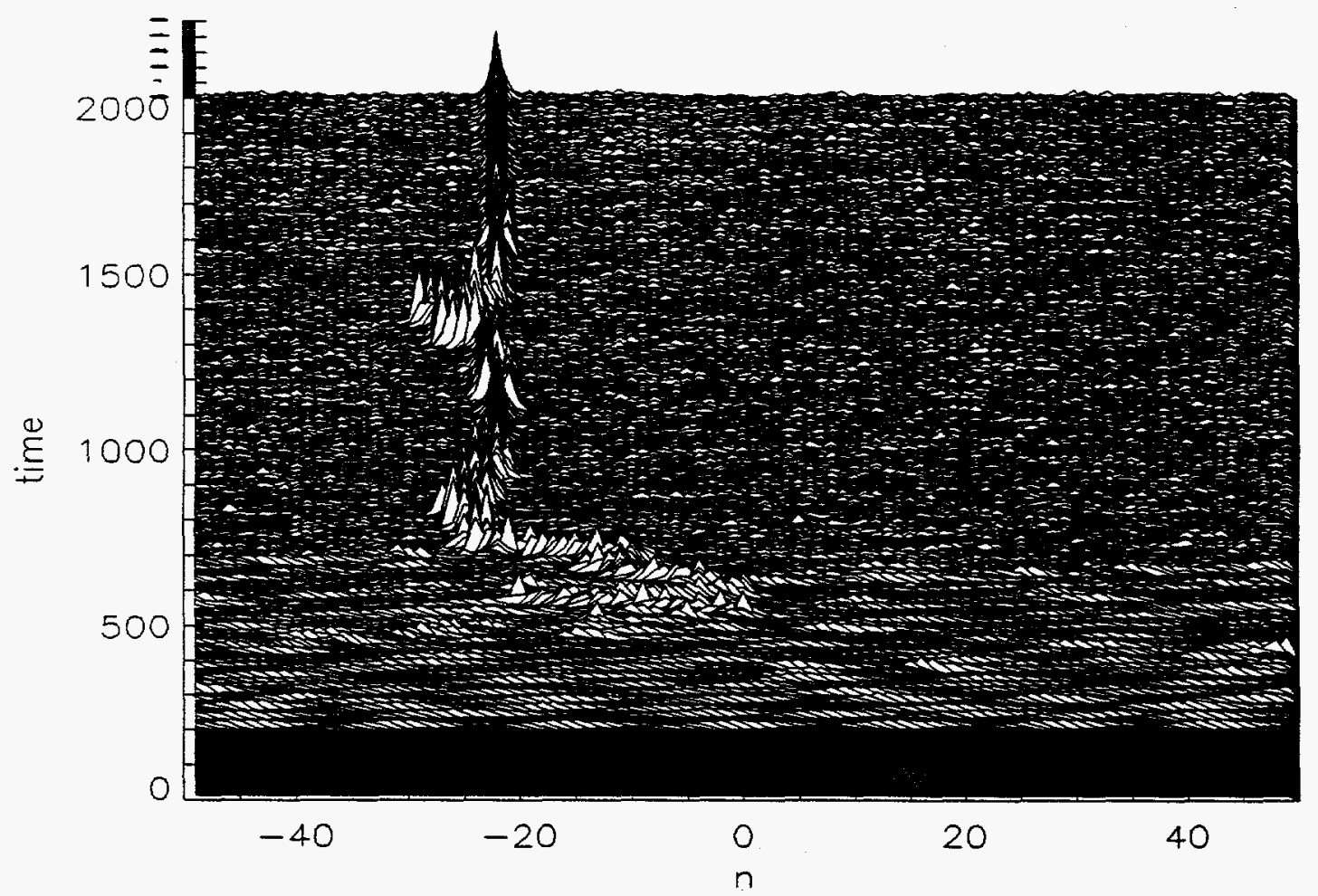

Figure 8: Localization: Evolution of the system (6) with $\mu=1$ and $\nu=0.2$ (nonintegrable). The initial "black sea" is due to the fact that the system still stays very close to the initial condition. $\left|\psi_{n}(t)\right|$ is plotted here.

$x_{i}$, with $x_{i}$ the $i$ th zero of the zeroth order Bessel function. Fig. 4 shows the situation at resonance, where the dynamical localization is perfect: the $\delta$-function evolves into localized radiation in which the soliton performs exact periodic oscillations. Moving off resonance, as in Fig. 5, results in a slow (quasi-linear) dispersion of the radiation. It is important to appreciate that the dynamical localization and Bloch oscillations are a property of lattice discreteness and not peculiar to the special integrable limit. Thus, for example, Fig. 6 shows the evolution with $\mu=0, \nu=1$. We see that, with the same initial condition as in Fig. 4, the soliton and radiation now interfere destructively. However, the dynamics remains bounded if the frequency $\omega$ is at resonance: This is essentially as expected for the linear case ${ }^{17}$. The dynamics in this nonintegrable case is probably chaotic but bounded (i.e., "localized chaos").

Finally, it is interesting to use the tunability of system (6) to study the the interplay of recurrence and stochasticity. In Figs. 7 and 8, we show the evolution of the unperturbed system (6) with periodic initial condition $\psi_{n}=A \exp (i k n)$, where $A=0.3$ and $k=10(2 \pi / N)$ and $N$ is the total number of lattice sites. In Fig. 7 $\nu=0$ (integrable) and in Fig. $8 \nu \neq 0$ (nonintegrable). In the integrable case, perfect recurrence is observed, whereas in Fig. 8 rapid self-focusing takes place to a localized structure whose center of mass may undergo intermittent dynamics between steady 


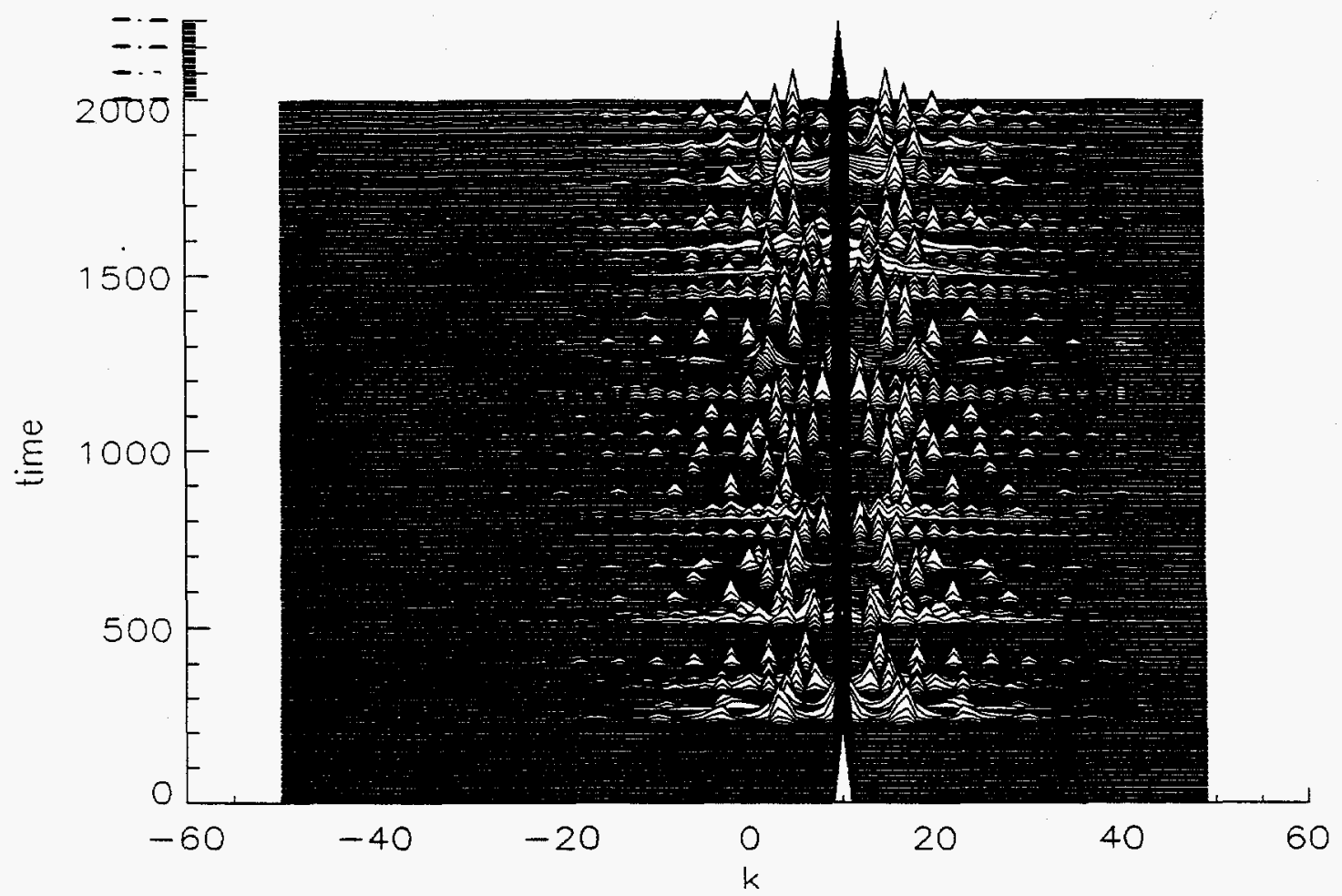

Figure 9: The time evolution of the spatial Fourier modes in the system (6) with $\mu=1$ and $\nu=0$ (integrable).

and irregular behavior. This corresponds to localization in wave-vector space (Fig. 9 ) versus stochasticization in wave-vector space (Fig. 10). All the simulations were carried out for the total run time $t=8000$ (time units). Clearly, these behaviors are important for the understanding of statistical mechanics of nonlinear lattices, and the tunability of system (6) makes it a teaching example deserving further study.

\section{A Quantum Polaron Model with Disorder}

Discrete NLS equations frequently appear in the literature as a consequence of adiabatic slaving of a fast field coupled (linearly) to a slow one. This is the case for plasmas, for Davydov-Scott "solitons", Holstein polarons, magnetic polarons, etc. However, when we wish to add disorder, thermal or quantum noise, we should be cautious in identifying with the simple addition of disorder or noise to the NLS equations derived in the absence of the perturbation - slaving, multiple-scale, rotating-wave derivations are adiabatic, and may result in additional space or time scales. One possible consequence for polarons generally is that we have to follow in detail the relative dynamics of the coupled fields as well as the center-of-mass motion of the composite particle-like bound state. That is, we have to include retardative, nonadiabatic effects, as in the case of Cherenkov radiation - phonon emission which limits the 


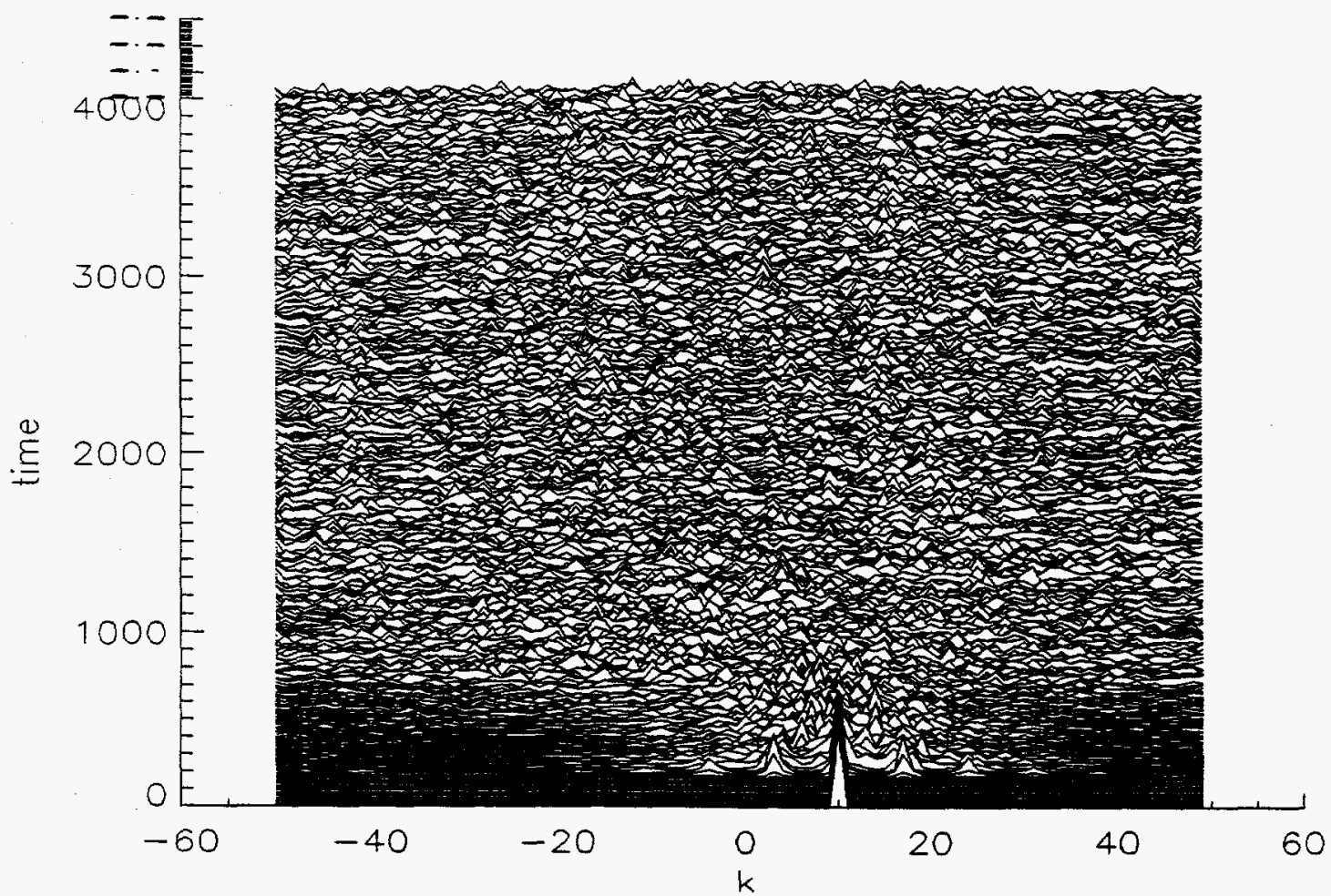

Figure 10: The time evolution of the spatial Fourier modes in the system (6) with $\mu=1$ and $\nu=0.2$ (nonintegrable).

mobility of polarons.

Here, we briefly describe a three-site model of electrons coupled to lattice vibrations which can be solved exactly numerically, including both electrons and phonons fully quantum-mechanically. In this way we can assess nonlinear and nonadiabatic effects systematically and also include the effects of disorder and temperature. Complete details are given in Refs. 19-21. In the following we briefly review some important aspects for our present purposes.

As a prototype of polaronic behavior, we consider a three-site model ${ }^{18,19}$ with two phonon modes, also known as a molecular-crystal Hamiltonian. It can be written in the form

$$
H=H_{\mathrm{el}}+H_{\mathrm{el}-\mathrm{ph}}+H_{\mathrm{ph}}
$$

where

$$
\begin{aligned}
H_{\mathrm{el}} & =\sum_{n} \epsilon_{n} \rho_{n}+U \sum_{n} \rho_{n \uparrow} \rho_{n \downarrow}+\sum_{\left\langle n n^{\prime}\right\rangle \sigma} t_{n n^{\prime}}\left(c_{n \sigma}^{\dagger} c_{n^{\prime} \sigma}+\text { h.c. }\right) \\
H_{\mathrm{el}-\mathrm{ph}} & =\lambda_{s}\left(a_{s}+a_{s}^{\dagger}\right)\left(\rho_{1}-\rho_{2}+\rho_{3}\right)+\lambda_{a}\left(a_{a}+a_{a}^{\dagger}\right)\left(\rho_{3}-\rho_{1}\right) \\
H_{\mathrm{ph}} & =\hbar \omega_{s} a_{s}^{\dagger} a_{s}+\hbar \omega_{a} a_{a}^{\dagger} a_{a} .
\end{aligned}
$$

Here, $c_{n \sigma}^{\dagger}$ creates an electron of spin $\sigma$ at site $n, \rho_{n \sigma}=c_{n \sigma}^{\dagger} c_{n \sigma}$, and $\rho_{n}=\sum_{\sigma} \rho_{n \sigma}$. The notation $\left\langle n n^{\prime}\right\rangle$ refers to the bonds and $t_{n n^{\prime}}$ is the hopping matrix element between 


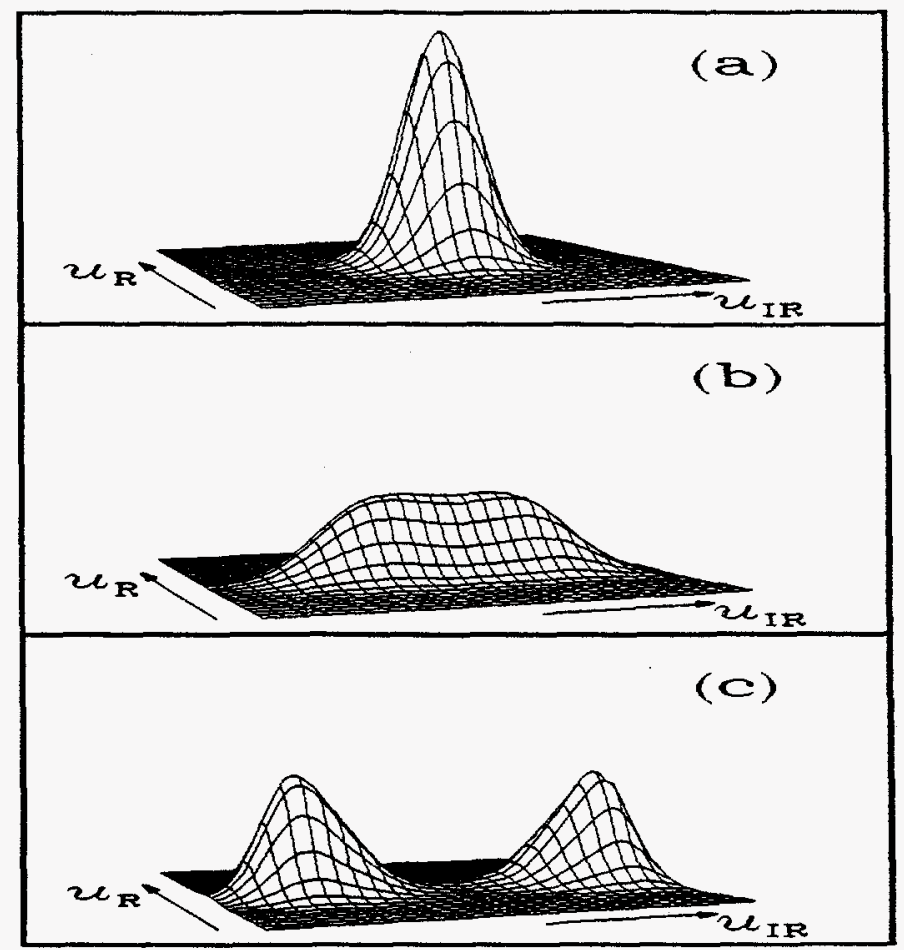

Figure 11: Probability distributions of Raman and infrared coordinates $u_{R}$ and $u_{I R}$ in (a) weak, (b) intermediate, and (c) strong coupling regimes of $\lambda_{I R}$.

sites $n$ and $n^{\prime}$. An on-site electron-electron interaction of strength $U$ is included for generality. The isolated three-site cluster has two phonon modes for displacements along the cluster axis that do not change the center of mass. These bare modes are assumed to be harmonic with well-defined parity. Usually, the symmetric and the antisymmetric phonon modes are referred to Raman and infrared active. These modes are described by boson operators $a_{s}$ and $a_{a}$ with bare frequencies $\omega_{s}$ and $\omega_{a}$, where $s(a)$ denotes the symmetric (antisymmetric) mode, respectively. The corresponding linear electron-phonon coupling constants are $\lambda_{s}$ and $\lambda_{a}(\geq 0)$.

Despite the above model's apparent simplicity, it can describe a wide variety of interesting phenomena, ranging from polaron formation and tunneling, to multipolaron interactions. Rather than considering all these aspects, we focus here on a polaron tunneling regime ${ }^{18}$. In this regime for sufficiently large values of $\lambda_{a}$, the model dynamically generates a new length scale associated with a double-well structure in the antisymmetric-phonon mode coordinate, $u_{a}=a_{a}+a_{a}^{\dagger}$ (Fig. 11). The motion of the lattice is strongly correlated with the electron motion, corresponding to polaron tunneling: the bare time-scale separation of electronic and phonon degrees of freedom ceases to exist. Furthermore, as the system's parameters move from the weak-coupling to the strong-coupling regime, a pair of symmetric and antisymmetric low-energy 


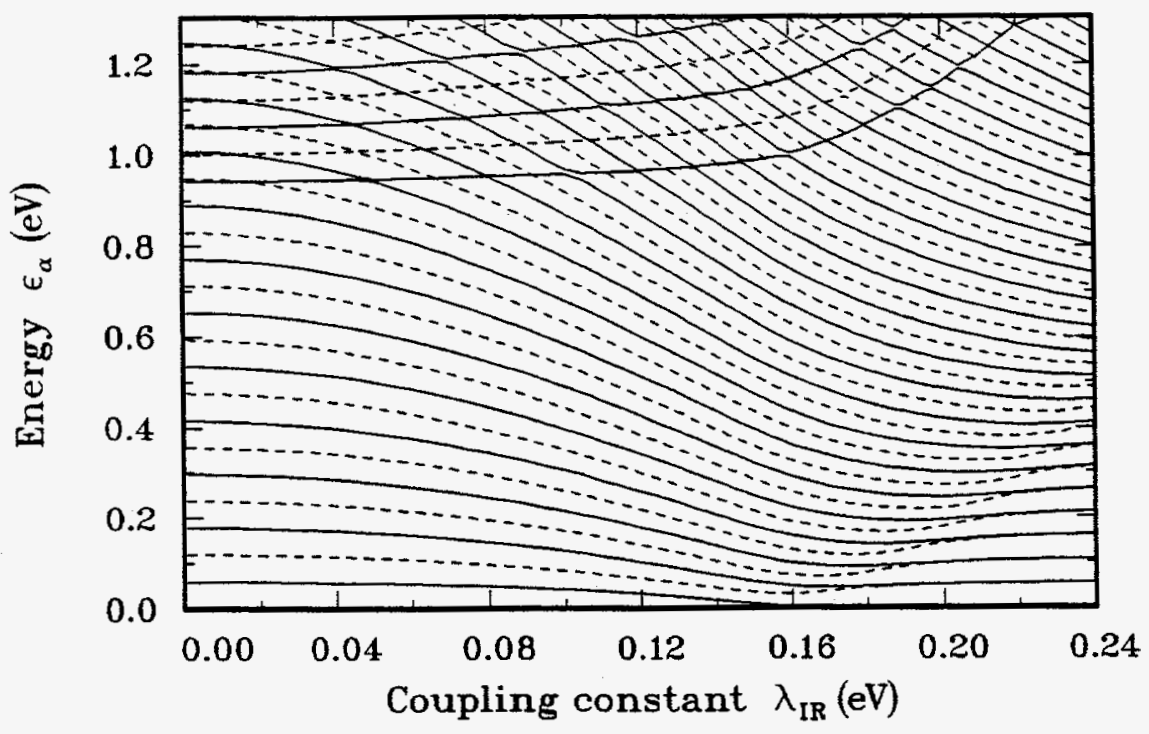

Figure 12: The low-energy spectrum of spin-singlet excited states relative to the ground state (even-parity) of the three-site cluster as a function of $\lambda_{I R}$ with $\lambda_{R}=0$ and no Raman excitations shown. The even- and odd-parity states are shown as dashed and solid lines, respectively. Note the onset of avoided level crossings at sufficiently large energies and $\lambda_{I R}$.

states become degenerate, signaling the appearance of the double well, and the lowenergy symmetric states show minima in the intermediate-coupling regime (onset of polaron tunneling), whereas the energies of the antisymmetric states show much weaker minima if at all ${ }^{19,20}$; see Fig. 12.

The polaron-tunneling problem is an exciting model to explore, because it not only behaves as a prototype for nonlinear and nonadiabatic dynamics, but can also be applied to understand how the correlated structural and electronic properties on a local level are related to measurable macroscopic quantities. Signatures of polarons can be probed through various correlation functions which measure structural and optical (absorption and fluorescence) properties of the system. Moreover, (energyresolved) dynamic structure factors can be used to probe the system in unique way as they allow one to correlate length and time scale information ${ }^{20}$.

To model disorder, we assume that the system has two electrons with opposite spins $\left(S_{z}=0\right)$ on a linear cluster, which is parametrized so that $t_{13}=0, t_{12}=t$, $t_{23}=t+\delta, \epsilon_{1,3}=\epsilon_{o} \pm \Delta / 2$, and $\epsilon_{2}=0$. We also consider the parameter regime where $0 \leq t<\epsilon_{o}, U-\epsilon_{0}$. Large, positive values of the on-site electron-electron interaction, $U$, and large positive average energies of sites 1 and 3 , given by $\epsilon_{o}$, inhibit bipolaron formation at low energies and the low-energy physics is therefore characterized by polarons. The symmetry-breaking fields $\Delta$ and $\delta$ describe the disorder - diagonal and off-diagonal, respectively. These, as well as other parameters, can be varied so 


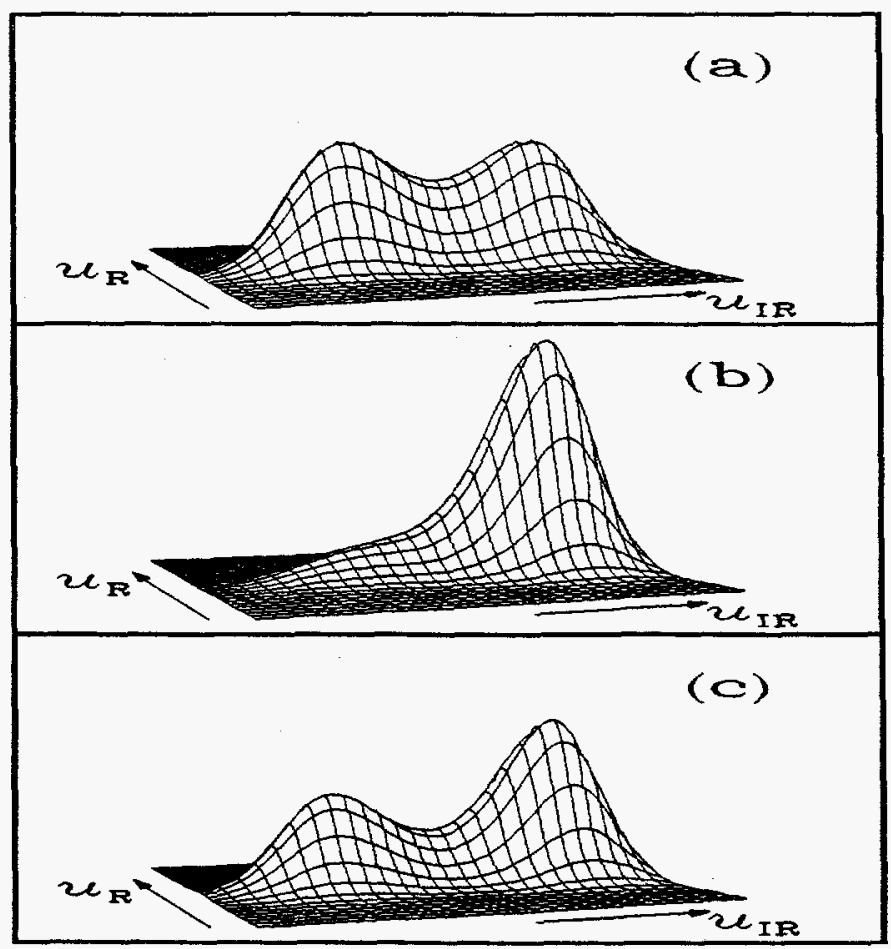

Figure 13: Probability distributions of Raman and infrared coordinates $u_{R}$ and $u_{I R}$ for (a) $\Delta=0$ and $T=0$, (b) $\Delta=2 \hbar \omega_{T}$ and $T=0$, and (c) $\Delta=2 \hbar \omega_{T}$ and $T=2 \hbar \omega_{T} / k_{B}$.

that the salient disorder-induced features can be studied transparently. Thus, we generally set $\lambda_{s}$ to zero, because the symmetric mode plays only a minor role in the polaron physics below.

In the following examples, we vary $\Delta$ and keep the other parameters constant. This approach allows us to study the interplay between localization due to (diagonal) disorder and polaronic nonlinearity due to (on-site) electron-phonon coupling. The interplay leads to a crossover at $\Delta \sim \hbar \omega_{T}^{20}$ : for $\Delta \lesssim \hbar \omega_{T}$, the system is in the polaron-tunneling regime, whereas, for $\Delta \gtrsim \hbar \omega_{T}$, the polaron is localized around site 3; see Fig. 13. In contrast to disorder, thermal fluctuations tend to delocalize the polaron: if we let $\Delta \sim \hbar \omega_{T}$, our calculations verify the intuitive expectation that the delocalization develops at temperatures $T \sim \Delta / k_{B}$. To monitor the stability and localization of the polaron, we consider two diagnostic quantities ${ }^{21}$.

First, we measure the correlation between the electrons and the phonon coordinate in the ground state. Defining deviations of the operators as $\delta O=O-\langle O\rangle$ and denoting $\rho_{13}=\rho_{1}-\rho_{3}$, we introduce the correlation factor

$$
\mathcal{R}=\frac{\left\langle\delta \rho_{13} \delta u_{a}\right\rangle}{\sqrt{\left\langle\left(\delta \rho_{13}\right)^{2}\right\rangle\left\langle\left(\delta u_{a}\right)^{2}\right\rangle}}
$$




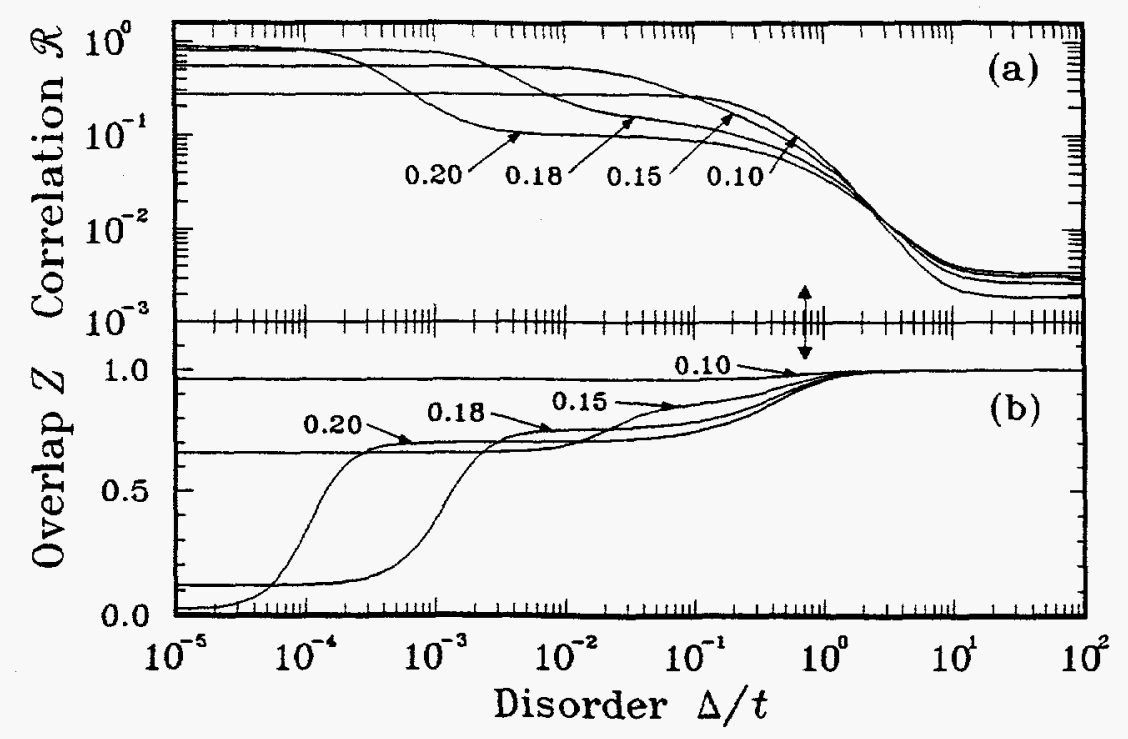

Figure 14: (a) The correlation factor $\mathcal{R}$ and (b) the ground-state overlap factor $Z$ as a function of the diagonal disorder parameter $\Delta$ for $U / t=16$ and for the electron-phonon coupling constant $\lambda_{a} / t$ equal to $0.10,0.15,0.18$, and 0.20 . In the absence of disorder, the corresponding tunneling energies $\epsilon_{T} / t$ are $5.3 \times 10^{-2}, 1.5 \times 10^{-2}, 1.2 \times 10^{-3}$, and $1.1 \times 10^{-4}$, respectively. The double-headed arrow marks the energy scale $w$, which is the bare electron bandwidth for the lowest energy "band" (i.e., in the absence of electron-phonon interactions). The other parameters are $\epsilon_{o} / t=4, \hbar \omega_{a} / t=0.08, \lambda_{s}=0$.

Although the expectation values may be taken relative to any state, we consider here only the ground-state expectation values which will describe the polaronic state. The closer $\mathcal{R}$ is to unity, the more correlated are the electron and phonon dynamics, implying strong polaronic binding of the electron to its phonon cloud. In contrast, $\mathcal{R}=0$ signals non-polaronic behavior, where the motions of electrons and phonons are completely decoupled.

Second, we consider the dressing of electrons by phonons in terms of the overlap factors between the ground states of the system in the absence and in the presence of the electron-phonon interaction. For the ground states, the polaronic dressing of electrons is conveniently defined by the overlap factor,

$$
Z=\left|\left\langle\Psi_{o}\left(\lambda_{a}=0, \Delta\right) \mid \Psi_{o}\left(\lambda_{a}, \Delta\right)\right\rangle\right|^{2}
$$

where $\left|\Psi_{o}\left(\lambda_{a}, \Delta\right)\right\rangle$ is the ground state of the system for the shown parameters. $Z=1$ signifies no phonon dressing, and decreasing $Z$ implies increasing dressing.

Here we consider only diagonal disorder as an example, because it is conceptually easier to understand; off-diagonal disorder has been studied elsewhere ${ }^{21}$. Our results for diagonal disorder are summarized in Fig. 14, which shows $\mathcal{R}$ and $Z$ as a function 
of the disorder strength $\Delta$ for $U / t=16$ and for various values of the electron-phonon coupling constant $\lambda_{a}{ }^{21}$. Both $\mathcal{R}$ and $Z$ exhibit a crossover behavior at a larger value of $\Delta$ and another one for a smaller value when $\lambda_{a}$ is large enough that the system is in the polaron tunneling regime. These results can be understood in terms of two characteristic energy scales: the polaron tunneling energy, $\epsilon_{T}$, and $w$, the energysplitting of the lowest-energy spin-singlet states (with opposite parity), which plays the role of the lowest-energy bare electron bandwidth in our finite cluster. For $U / t=$ 16 and in the absence of disorder, $w / t=0.72$. As the symmetry-breaking disorder field $\Delta$ is increased, the first crossover at $\Delta \sim \epsilon_{T}$ describes the localization of the polaron to essentially one site. As $\Delta$ is increased further so that $\Delta \sim w, \mathcal{R}$ decreases sharply to a small value and $Z$ approaches unity. This signals the breaking of the composite character of the polaron, so that the electron is no longer coherently dressed by phonons - a distinctly nonadiabatic phenomenon.

\section{Discussion}

The purpose of the examples described briefly in this article was to illustrate some of the fascinating interplays between nonlinearity, nonadiabaticity, disorder, noise (thermal, quantum or otherwise) and lattice discreteness, leading to many instances of competing length-scales and time-scales. Clearly, there are many questions left unanswered and indications for future research. These include:

(i) Merging the fields of "nonlinearity" and "Nonequilibrium statistical mechanics". Evidently, the stochastic fluctuations and disorder must be considered along with nonlinearity in many natural phenomena occurring in solids, fluids, biological processes, etc. We need to identify broad classes of behavior, including both intra- and inter-attractor dynamics. As in "glassy" systems generally, this results in a spectrum of fast and slow time-scales, respectively.

(ii) Understanding the ranges of validity of stationary-phase and collective coordinate nonlinear mode reduction approximations for particle-like nonlinear structure in the presence of perturbations, including qualitatively new discrete lattice effects. Equally importantly, we must understand how noise/disorder competes with the formation of particle-like structures (e.g., inhibits collapse/self-focusing/blow-up). Here it is important to appreciate that stationary states completely describe the evolution of linear Schrödinger equations with stationary noise; however, in the nonlinear case there are (generally) not only multiple scaling variables characterized by linear, disordered and nonlinear, ordered basins of attraction, but also "intermittent" solutions which visit a wider solution space.

(iii) Understanding the ranges of validity of nonlinear Schrödinger-like descriptions of "polarons", whether in the context of electron-phonon, exciton-phonon, or magnonphonon coupling. Since NLS descriptions are based on adiabatic slaving, multiple time-scale, rotating wave, etc., approximations, adding disorder or noise (thermal or quantum) requires that we systematically include these new space/time scales 
and not add them in ad hoc fashion to the bare NLS equation - "adiabaticity" must be defined very carefully in such multiple time-scale situations. In particular, quantum lattice effects may be qualitatively imp tant - as in the example described in Section 4. It is important that this not be left as an abstract mathematical physics issue - physically observable properties (optical, structural, transport, etc.) must be evaluated, because these are particular integrations of eigenenergy and eigenfunction structure which may smooth out many detailed quantum features and restore the effective validity of suitable approximations.

\section{References}

1. R. Scharf, Yu.S. Kivshar, A. Sánchez, A.R. Bishop, Phys. Rev. A45 (1992) R5369.

2. A. Sánchez, R. Scharf, A.R. Bishop, and L. Vázquez, Phys. Rev. A45 (1992) 6031.

3. A. Sánchez, A.R. Bishop, and F. Domínguez-Adame, Phys. Rev. E49 (1994) 4603.

4. R. Scharf and A.R. Bishop, Phys. Rev. A46 (1992) 2973.

5. R. Scharf and A.R. Bishop, Phys. Rev. E47 (1993) 1375.

6. M.J. Ablowitz and J.F. Ladik, J. Math. Phys., 17 (1976) 1011.

7. D. Cai, A.R. Bishop, and N. Grønbech-Jensen, Phys. Rev. Lett. 72 (1994) 591.

8. M. Salerno, Phys. Rev. A46 (1992) 6856.

9. P. Marguié, J.M. Bilbault and M. Remoissenet, Preprint (1994).

10. D. Cai, Ph.D thesis "Structure and Dynamics of Excitations in Discrete Nonlinear Schrödinger Systems.

11. S. Takeno, J. Phys. Soc. Jpn, 61 (1992) 2821; A.J. Sievers and S. Takeno, Phys. Rev. Lett. 61 (1988) 970; these proceedings.

12. R. Scharf and A.R. Bishop, Phys. Rev. A43 (1991) 6535.

13. H. Chen and C. Liu, Phys. Rev. Lett. 37 (1976) 693.

14. V.V. Konotop, O.A. Chubykalo and L. Vázquez, Phys. Rev. E48 (1993) 563.

15. M. Bruschi, D. Levi, and O. Ragnisco, Nuovo Cimento, 53A (1979) 21.

16. D. Cai, A.R. Bishop, N. Grønbech-Jensen, and M. Salerno, Preprint LA-UR-942026.

17. D.H. Dunlap and V.M. Kenkre, Phys. Lett. A 127 (1988) 438; Phys. Rev. B34 (1986) 3625.

18. J.Mustre de Leon, I. Batistic, A.R. Bishop, S. Conradson, and S. Trugman, Phys. Rev. Lett. 68, (1992) 3236.

19. M. Salkola, A.R. Bishop, J. Mustre de Leon, and S. Trugman, Phys. Rev. B49, (1994) 3671.

20. M. Salkola, A.R. Bishop, S. Trugman, and J. Mustre de Leon, preprint (1994).

21. M. Spicci, M. Salkola, and A.R. Bishop, J. Phys. Condens. Matter 6, (1994) L361. 